manuscript No.

(will be inserted by the editor)

\title{
Optimality conditions in convex multiobjective $\mathrm{SIP}^{\star}$
}

\author{
Miguel A. Goberna ${ }^{1}$, Nader Kanzi ${ }^{2}$ \\ 1 Department of Mathematics, University of Alicante, Alicante, Spain. e-mail: \\ mgoberna@ua.es \\ 2 Department of Mathematics, Payame Noor University (PNU), Tehran, Iran. \\ e-mail: nad.kanzi@gmail.com
}

The date of receipt and acceptance will be inserted by the editor

\begin{abstract}
The purpose of this paper is to characterize the weak efficient solutions, the efficient solutions, and the isolated efficient solutions of a given vector optimization problem with finitely many convex objective functions and infinitely many convex constraints. To do this, we introduce new and already known data qualifications (conditions involving the constraints and/or the objectives) in order to get optimality conditions which are expressed in terms of either Karusk-Kuhn-Tucker multipliers or a new gap function associated with the given problem.
\end{abstract}

\section{Introduction}

An optimization problem

$(P) \quad$ minimize $f(x):=\left(f_{1}(x), \ldots, f_{p}(x)\right)$ subject to $g_{t}(x) \leq 0, t \in T,(1)$

with real-valued objective functions $f_{i}: \mathbb{R}^{n} \longrightarrow \mathbb{R}, i \in I:=\{1, \ldots, p\}$, and extended real-valued constraint functions $g_{t}: \mathbb{R}^{n} \longrightarrow \overline{\mathbb{R}}:=[-\infty,+\infty]$, $t \in T$, is called scalar when $p=1$ and multiobjective when $p \geq 2$, it is called ordinary when $T$ is finite and semi-infinite otherwise, and it is called convex (linear, etc.) when all the involved functions (called the data) are convex (resp. linear, etc.). The Euclidean spaces $\mathbb{R}^{n}$ and $\mathbb{R}^{p}$ are called decision space and objective (or outcome) space, respectively.

The objective of this paper is to characterize different types of solutions for a consistent convex multiobjective semi-infinite programming (MOSIP

^ This research was partially cosponsored by the Ministry of Economy and Competitiveness (MINECO) of Spain, and by the European Regional Development Fund (ERDF) of the European Commission, Project MTM2014-59179-C2-1-P. 


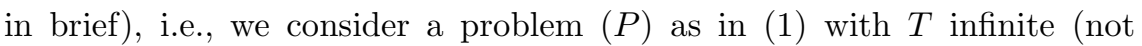
necessarily equipped with some topology), $p \geq 2$, and convex data. This type of problem arises in a natural way in robust convex multiobjective programming when at least one of the uncertainty sets for the constraints is infinite (see 24] and references therein). We also assume that all constraint functions are lower semicontinuous (lsc for short). So, the feasible set of $(P)$, denoted by $S$, is a nonempty closed convex set. The characterization of the solutions of $(P)$ in this paper are expressed either in terms of multipliers or in terms of an associated scalar function called gap function.

Many works have been published on optimality conditions in ordinary multiobjective convex programming and in scalar semi-infinite programming via data qualifications (DQs in short), called constraint qualifications (CQs) and objective qualifications (OQs) when they exclusively involve the constraints and the objectives, respectively (see, e.g., [18, [14, 25], 27, etc.). The closest antecedents for this paper are the following works dealing with Karush-Kuhn-Tucker (KKT in short) type optimality conditions for different classes of multiobjective semi-infinite programming problems:

- 12, on linear MOSIP problems.

- 13, where the data are convex and satisfy the assumption that $(P)$ is continuous, meaning that $T$ is a compact Hausdorff topological space and the mapping $(t, x) \longmapsto g_{t}(x)$ is continuous (and so finite-valued) on $T \times \mathbb{R}^{n}$. This is the case whenever $T$ is a finite set equipped with the discrete topology and the constraint functions are finite-valued. The continuity of $(P)$ is a strong assumption allowing to get KKT conditions via the linearization of the data.

- 16, where the data are differentiable.

- 6], 7], 20], 21], and [23, where the data are locally Lipschitz on $\mathbb{R}^{n}$, assumption which allows to apply the non-smooth analysis machinery.

Observe that a proper convex function is subdifferentiable (but not necessarily differentiable) at the interior of its domain, while it is also locally Lipschitz on that set under some additional assumption (e.g., when it is bounded above on some nonempty open subset of its domain [33. Corollary 2.2.13]). So, the class of MOSIP problems considered in [12] is a subclass of those considered in [16, 6], 77, 20, 21, and [23, while [13] does not, except in particular cases (e.g., when all data are bounded above on $\mathbb{R}^{n}$ ). Notice also that the class of MOSIP problems in 13 is much smaller than the one considered in this paper. In conclusion, to the best of the authors' knowledge, this is the first paper providing optimality conditions for noncontinuous convex MOSIP problems.

Concerning the second tool used in this paper to get optimality conditions, let us recall that the term 'gap function' was the name given in 2 to the objective function of the reformulation of certain variational inequality as a scalar optimization unconstrained problem. By extension, this is the name usually given to those functions allowing to reformulate a given optimization problem as a simpler one. Gap functions have been proposed 
for scalar optimization problems with smooth convex data [17, ordinary multiobjective problems with differentiable data [5, ordinary multiobjective problems with nonsmooth data ([29, 31], 30]), scalar semi-infinite problems with quasiconvex data 22, etc. In this paper we introduce a new gap function in order to get optimality conditions in convex MOSIP.

We analyze in this paper three types of optimal solutions for $(P)$, namely:

- Weak efficient solutions, which are commonly considered those solutions that can be most easily computed. A recent result on vector optimization problems (where the decision and the objective spaces are possibly infinite dimensional) proved in [8, will be used to characterize weak solutions of $(P)$ in some particular cases.

- Efficient solutions, which are the most used in practice.

- Isolated efficient solutions, which are the most stable under small perturbations of the objective functions, as we comment after its definition below.

The definitions of efficient and weak efficient solutions involve orderings in $\mathbb{R}^{p}$ induced by its positive cone $\mathbb{R}_{+}^{p}$ and by its interior $\mathbb{R}_{++}^{p}$. Indeed, given $x, y \in \mathbb{R}^{p}$, we write $x \leqq y$ (resp. $x<y$ ) when $x_{i} \leq y_{i}\left(\operatorname{resp} . x_{i}<y_{i}\right)$ for all $i \in I$. Moreover, we write $x \leq y$ when $x \leqq y$ and $x \neq y$.

An element $\widehat{x} \in S$ is said to be an efficient solution (resp. a weak efficient solution) for $(P)$ if there is no $x \in S$ satisfying $f(x) \leq f(\widehat{x})$ (resp., $f(x)<$ $f(\widehat{x}))$. Moreover, following [10, we say that an element $\widehat{x} \in S$ is an isolated efficient solution for $(P)$ if there exists a constant $\nu>0$ such that

$$
\max _{i \in I}\left\{f_{i}(x)-f_{i}(\widehat{x})\right\} \geq \nu\|x-\widehat{x}\|, \quad \forall x \in S .
$$

The weak efficient solutions and the efficient solutions have been characterized in [13] for convex continuous MOSIP problems, while it seems that no previous work deals with isolated efficient solution in the convex setting. Obviously, any isolated efficient solution is an efficient solution (actually the unique efficient solution) and any efficient solution is a weak efficient solution. The concept of isolated efficient solution is an extension, to $p>1$, of the concept of isolated local minimum introduced in [3] to study the stability of scalar optimization problems. The isolated minima are called strongly unique optimal solutions in the linear semi-infinite setting, where they also play a crucial role in the sensitivity analysis with respect to perturbations of the objective function ([1], 15]). We now sketch the role that isolated efficient solutions could play in the MOSIP setting.

Assume that $\widehat{x}$ is an isolated efficient solution of $(P)$ with associated constant $\nu$. If we perturb the objective function $f_{i}$ with a function $p_{i}$ : $S \longrightarrow \mathbb{R}$ which is locally Lipschitz at $\widehat{x}$ with constant $L_{i}<\nu, i \in I$, and define $L:=\max _{i \in I} L_{i}<\nu$, then we have

$$
\begin{aligned}
\max _{i \in I}\left\{\left(f_{i}+p_{i}\right)(x)-\left(f_{i}+p_{i}\right)(\widehat{x})\right\} & \geq \max _{i \in I}\left\{\left[f_{i}(x)-f_{i}(\widehat{x})\right]-L_{i}\|x-\widehat{x}\|\right\} \\
& \geq \max _{i \in I}\left\{\left[f_{i}(x)-f_{i}(\widehat{x})\right]\right\}-L\|x-\widehat{x}\| \\
& \geq(\nu-L)\|x-\widehat{x}\|
\end{aligned}
$$


for any $x \in S$, so that $\widehat{x}$ is still an isolated efficient solution of the perturbed MOSIP problem

minimize $\left(f_{1}(x)+p_{1}(x), \ldots, f_{p}(x)+p_{p}(x)\right)$ subject to $g_{t}(x) \leq 0, t \in T$.

In the particular case of linear perturbations of the form $p_{i}(x)=c_{i}^{\prime} x$, with $c_{i} \in \mathbb{R}^{n}, i \in I$, we can consider the following parametric convex MOSIP problem,

$\left(P_{C}\right) \quad$ minimize $f(x)+C x$ subject to $g_{t}(x) \leq 0, t \in T$,

whose parameter is the matrix $C:=\left[c_{1}|\ldots| c_{p}\right]^{\prime} \in \mathcal{M}_{p \times n}$, the real linear space of $p \times n$ matrices equipped with any norm and null element $0_{p \times n}$ (the zero $p \times n$ matrix). In the same way that sensitivity analysis in scalar optimization deals with the optimal value function of a parametric (scalar) problem, the sensitivity analysis of $\left(P_{C}\right)$ is focused on the multifunction $\mathcal{F}: \mathcal{M}_{p \times n} \rightrightarrows \mathbb{R}^{p}$ associating to each $C \in \mathcal{M}_{p \times n}$ the image of the efficient set of $\left(P_{C}\right)$ by its objective function. If $\widehat{x}$ is an isolated efficient solution of $(P)$, then there exists a neighborhood $\mathcal{N}$ of $0_{p \times n}$ in $\mathcal{M}_{p \times n}$ where $\max _{i \in I}\left\|c_{i}\right\|<\nu$ for all $C=\left[c_{1}|\ldots| c_{p}\right]^{\prime} \in \mathcal{N}$. Since $\widehat{x}$ is the unique efficient solution of $\left(P_{C}\right)$ for any $C \in \mathcal{N}$, the restriction of $\mathcal{F}$ to $\mathcal{N}$ is the affine function $\mathcal{F}(C)=$ $f(\widehat{x})+C \widehat{x}$.

The paper is organized as follows. Section 2 introduces the necessary notations and concepts, and analyzes the relationships between the data qualifications used in this paper and other well known ones. The remaining three sections provide different characterizations of weakly efficient solutions (Section 3), efficient solutions (Sections 4), and isolated efficient solutions (Section 5) via KKT-type conditions, gap function, and continuous linear functionals on the constraint space (only in Section 3).

\section{Data qualifications}

Our notation and terminology are basically standard. Given a locally convex Hausdorff topological vector space $Z$ and a set $\emptyset \neq M \subseteq Z$, the closure of $M$, the interior of $M$, the relative interior of $M$, the strong quasi-relative interior of $M$, the boundary of $M$, the linear span of $M$, the convex hull of $M$, and the convex cone (containing the origin) generated by $M$ are respectively denoted by $\bar{M}, \operatorname{int}(M), \operatorname{ri}(M), \operatorname{sqri}(M), \operatorname{bd}(M), \operatorname{span}(M)$, $\operatorname{conv}(M)$, and cone $(M)$. The null vector of $Z$ is denoted by $0_{Z}$ and the topological dual of $Z$ is denoted by $Z^{*}$, always equipped with the weak* topology. The duality product of $z^{*} \in Z^{*}$ by $z \in Z$ is denoted by $\left\langle z^{*}, z\right\rangle$. In this paper we handle three infinite dimensional spaces related with the index set $T$ :

- $\mathbb{R}^{T}$ is the space of real-valued functions on $T$ equipped with the product topology. The support of $\lambda \in \mathbb{R}^{T}$ is $\left\{t \in T \mid \lambda_{t}:=\lambda(t) \neq 0\right\}$. 
$-l_{\infty}(T)$ is the Banach space of all bounded functions from $T$ to $\mathbb{R}$ equipped with the supremum norm.

- When $T$ is a compact topological space, $\mathcal{C}(T)$ represents the subspace of $l_{\infty}(T)$ formed by the continuous functions.

When $Z=\mathbb{R}^{n}$, the duality product $\langle x, y\rangle$ coincides with the scalar product $x^{\prime} y$, for $x, y \in \mathbb{R}^{n},\|x\|=\sqrt{x^{\prime} x}$ denotes the Euclidean norm of $x, \mathbb{B}_{n}$ represents the closed unit ball and $0_{n}$ stands for the null vector $0_{n}:=0_{\mathbb{R}^{n}}$. Given $A, B \subseteq \mathbb{R}^{n}$, one says that $A$ is closed regarding $B$ if $B \cap \bar{A}=B \cap A$.

The negative polar cone and the strictly negative polar cone of $\emptyset \neq M \subset$ $\mathbb{R}^{n}$ (not necessarily a convex cone) are defined respectively by $M^{0}:=\{d \in$ $\left.\mathbb{R}^{n} \mid x^{\prime} d \leq 0, \forall x \in M\right\}$, and $M^{-}:=\left\{d \in \mathbb{R}^{n} \mid x^{\prime} d<0, \forall x \in M\right\}$. The bipolar theorem (see, e.g., [18, Proposition III.4.2.7]) states that $\left(M^{0}\right)^{0}=$ $\overline{\text { cone }}(M)$. Also, it is well-known that if $M^{-} \neq \emptyset$, then $\overline{M^{-}}=M^{0}$.

Regarding extended functions, given $h: \mathbb{R}^{n} \longrightarrow \overline{\mathbb{R}}:=\mathbb{R} \cup\{ \pm \infty\}$, dom $h$ denotes the domain of $h$ and, for $x \in h^{-1}(\mathbb{R}), h^{\prime}(x ; d)$ and $\partial h(x)$ denote the directional derivative of $h$ at $x$ in the direction of $d \in \mathbb{R}^{n}$ and the Fenchel-Moreau subdifferential of $h$ at $x$, respectively. By $\Gamma\left(\mathbb{R}^{n}\right)$ we denote the class of all lsc, convex, and proper functions from $\mathbb{R}^{n}$ to $\overline{\mathbb{R}}$.

Given $\widehat{x} \in M \subseteq \mathbb{R}^{n}$, we denote the contingent cone (also called Bouligand tangent cone) and the feasible direction cone of $M$ at $\widehat{x}$, respectively, by $C(M, \widehat{x})$ and by $D(M, \widehat{x})$, i.e.,

$$
C(M, \widehat{x}):=\left\{v \in \mathbb{R}^{n} \mid \exists t_{r} \downarrow 0, \exists v_{r} \rightarrow v: \widehat{x}+t_{r} v_{r} \in M, \forall r \in \mathbb{N}\right\},
$$

and

$$
D(M, \widehat{x}):=\left\{d \in \mathbb{R}^{n} \mid \exists \delta>0: \widehat{x}+\varepsilon d \in M, \forall \varepsilon \in(0, \delta)\right\} .
$$

If $M$ is a closed convex set, then $\overline{D(M, \widehat{x})}=C(M, \widehat{x})$ for all $\widehat{x} \in M$ [18, Proposition III.5.2.1]. Then, the normal cone to $M$ at $\widehat{x}$ is $N(M, \widehat{x}):=$ $D^{0}(M, \widehat{x})=C^{0}(M, \widehat{x})$.

We associate with $(P)$ the following three functions:

- The supremum (or marginal) function $\psi: \mathbb{R}^{n} \longrightarrow \overline{\mathbb{R}}$ such that

$$
\psi(x):=\sup _{t \in T} g_{t}(x) .
$$

This lsc proper convex function allows to reformulate $(P)$ as an ordinary multiobjective programming problem with a single constraint:

$$
\left(P_{1}\right) \quad \text { minimize } f(x):=\left(f_{1}(x), \ldots, f_{p}(x)\right) \text { subject to } \psi(x) \leq 0 \text {. }
$$

- The infimum function $\iota: \mathbb{R}^{n} \longrightarrow \overline{\mathbb{R}}$ such that

$$
\iota(x):=\inf _{t \in T} g_{t}(x) .
$$


- The gap function $\vartheta: \mathbb{R}^{n} \times \prod_{i=1}^{p} \partial f_{i}(x) \times \Delta_{+}^{p} \longrightarrow \overline{\mathbb{R}}$, such that

$$
\vartheta(x, \xi, \lambda):=\sup _{y \in S}\left\{\sum_{i=1}^{p} \lambda_{i} \xi_{i}^{\prime}(x-y)\right\}
$$

with $\Delta_{+}^{p}:=\left\{\left(\alpha_{1}, \ldots, \alpha_{p}\right) \in \mathbb{R}_{+}^{p} \mid \sum_{i=1}^{p} \alpha_{i}=1\right\}$. In the particular case that $p=1, \lambda=1$, and $f$ is a convex differentiable function, $\vartheta$ coincides with the gap function introduced in 17 .

We also associate with $(P)$ and $\widehat{x} \in S$ the following sets:

- The sublevel sets of $\widehat{x}$ w.r.t. the objectives,

$$
Q^{i}(\widehat{x}):=\left\{x \in S \mid f_{l}(x) \leq f_{l}(\widehat{x}), \forall l \in I \backslash\{i\}\right\}, i \in I,
$$

with the convention that $Q^{1}(\widehat{x})=S$ in the scalar case $(p=1)$.

- The set of $\varepsilon$-active indices at $\widehat{x}$, with $\varepsilon>0$,

$$
T_{\varepsilon}(\widehat{x}):=\left\{t \in T \mid-\varepsilon \leq g_{t}(\widehat{x})\right\} .
$$

- The set of active indices at $\widehat{x}, T(\widehat{x}):=T_{0}(\widehat{x})$, i.e., $T(\widehat{x})=\{t \in T \mid$ $\left.g_{t}(\widehat{x})=0\right\}$.

- The convex hull of the set of subgradients at $\widehat{x}$ of the objective functions:

$$
F_{*}(\widehat{x}):=\operatorname{conv}(F(\widehat{x})), \text { where } F(\widehat{x}):=\bigcup_{i=1}^{p} \partial f_{i}(\widehat{x}) .
$$

- The convex conical hull of the set of subgradients at $\widehat{x}$ of the active constraints at $\widehat{x}$ :

$$
G_{*}(\widehat{x}):=\text { cone }(G(\widehat{x})), \text { where } G(\widehat{x}):=\bigcup_{t \in T(\widehat{x})} \partial g_{t}(\widehat{x}) .
$$

If $t \in T(\widehat{x}), \xi \in \partial g_{t}(\widehat{x})$, and $d \in D(S, \widehat{x})$, there exists $\delta>0$ such that $\widehat{x}+\delta d \in S$ and, so, $0 \geq g_{t}(\widehat{x}+\delta d) \geq \delta \xi^{\prime} d$. Since $\xi^{\prime} d \leq 0$ for all $d \in D(S, \widehat{x}), \xi \in D^{0}(S, \widehat{x})=N(S, \widehat{x})$. Thus, $G(\widehat{x}) \subseteq N(S, \widehat{x})$ and taking convex hulls in both sides we get

$$
G_{*}(\widehat{x}) \subseteq N(S, \widehat{x}) .
$$

We consider in this paper two global CQs (i.e., CQs not involving $\widehat{x} \in S$ ), nine local CQs, two local data qualifications (DQs in brief, i.e., conditions involving all data), and one objective qualification (OQ) for our given convex MOSIP problem $(P)$. Most of these conditions are introduced (or at least inspired in conditions used) in works mentioned in Section 1:

- The Slater $C Q$ (SCQ, [13) holds when there is a $x_{0} \in \mathbb{R}^{n}$ (called Slater point) such that $g_{t}\left(x_{0}\right)<0$ for all $t \in T$. 
- The strong Slater $C Q$ (SSCQ, 12]) holds when there exist $x_{0} \in \mathbb{R}^{n}$ (called strong Slater point) and $\varepsilon>0$ (slack) such that $g_{t}\left(x_{0}\right) \leq-\varepsilon$, for all $t \in T$. Obviously, $(P)$ satisfies SSCQ if and only if $\left(P_{1}\right)$ satisfies SCQ.

- The Mangasarian-Fromovitz $C Q$ (MFCQ, 19]) holds at $\widehat{x}$ if $G(\widehat{x}) \neq \emptyset$ and $G^{-}(\widehat{x}) \neq \emptyset$, otherwise (this property is also called local Slater CQ in 13 ).

- The perturbed Mangasarian-Fromovitz $C Q$ (PMFCQ, 27]) holds at $\widehat{x} \in$ $S$ if there exists $x_{*} \in \mathbb{R}^{n}$ such that

$$
\inf _{\varepsilon>0} \sup \left\{\xi^{\prime} x_{*} \mid \xi \in \bigcup_{t \in T_{\varepsilon}(\widehat{x})} \partial g_{t}(\widehat{x})\right\}<0 .
$$

(This CQ has been recently introduced by Mordukhovich and Nghia in the framework of non-continuous smooth scalar semi-infinite programming.)

- The local Farkas-Minkowski $C Q$ (LFMCQ, [14]) holds at $\widehat{x} \in S$ when $N(S, \widehat{x})=G_{*}(\widehat{x})$.

- The Cottle CQ (COCQ) holds at $\hat{x}$ when $\left\{d \in \mathbb{R}^{n} \mid \psi^{\prime}(\hat{x} ; d)<0\right\} \neq \emptyset$.

- The Kuhn-Tucker $C Q$ (KTCQ, 13]) at $\widehat{x} \in S$ when

$$
\left\{d \in \mathbb{R}^{n} \mid \psi^{\prime}(\widehat{x} ; d) \leq 0\right\} \subseteq C(S, \widehat{x}) .
$$

(Since $S$ is convex, we have replaced, at the definition of KTCQ given in [13, page 35], the cone of attainable directions of $S$ at $\widehat{x}$ by $C(S, \widehat{x})$.)

- The Pshenichnyi-Levin-Valadier CQ (PLVCQ, 25]) holds at $\widehat{x}$ when $\partial \psi(\widehat{x}) \subseteq G_{*}(\widehat{x})$.

- The closed cone $C Q$ (CCCQ) holds at $\widehat{x}$ when $G_{*}(\widehat{x})$ is closed.

- The Abadie $C Q$ (ACQ, 25]) holds at $\widehat{x} \in S$ when $G(\widehat{x}) \neq \emptyset$ and $G^{0}(\widehat{x}) \subseteq$ $C(S, \widehat{x})$.

- The weak Abadie $D Q$ (WADQ, [14]) holds at $\widehat{x} \in S$ when $G(\widehat{x}) \neq \emptyset$ and $F^{-}(\widehat{x}) \cap G^{0}(\widehat{x}) \subseteq C(S, \widehat{x})$.

- The extended Abadie $D Q$ (EADQ, [26]) holds at $\widehat{x}$ when $G(\widehat{x}) \neq \emptyset$ and

$$
F^{0}(\widehat{x}) \cap G^{0}(\widehat{x}) \subseteq \bigcap_{i=1}^{p} C\left(Q^{i}(\widehat{x}), \widehat{x}\right) .
$$

- The Maeda OQ (MOQ, [26]) holds at $\widehat{x}$ when

$$
F^{0}(\widehat{x}) \subseteq\left\{0_{n}\right\} \cup \bigcup_{i=i}^{p}\left(\partial f_{i}(\widehat{x})\right)^{-} .
$$

The next result gives a checkable condition equivalent to the $M O Q$.

Proposition 1 The $M O Q$ holds at $\widehat{x} \in S$ if and only if $\operatorname{span}(F(\widehat{x}))=\mathbb{R}^{n}$. 
Proof Obviously, $\operatorname{span}(F(\widehat{x}))=\mathbb{R}^{n}$ if and only if cone $(F(\widehat{x}) \cup(-F(\widehat{x})))=$ $\mathbb{R}^{n}$ if and only if $\overline{\text { cone }}(F(\widehat{x}) \cup(-F(\widehat{x})))=\mathbb{R}^{n}$ or, equivalently, by the Farkas lemma for semi-infinite systems [14, Corollary 3.1.3],

$$
\left\{x \in \mathbb{R}^{n} \mid \xi^{\prime} x \leq 0, \forall \xi \in F(\widehat{x}) \cup(-F(\widehat{x}))\right\}=\left\{0_{n}\right\},
$$

i.e.,

$$
F^{0}(\widehat{x}) \cap\left(\left\{x \in \mathbb{R}^{n} \mid \xi^{\prime} x \geq 0, \forall \xi \in F(\widehat{x})\right\} \backslash\left\{0_{n}\right\}\right)=\emptyset .
$$

We finally reformulate (5) as

$$
\begin{aligned}
F^{0}(\widehat{x}) & \subseteq \mathbb{R}^{n} \backslash\left(\left\{x \in \mathbb{R}^{n} \mid \xi^{\prime} x \geq 0, \forall \xi \in F(\widehat{x})\right\} \backslash\left\{0_{n}\right\}\right) \\
& \subseteq\left\{0_{n}\right\} \cup\left[\mathbb{R}^{n} \backslash\left(\left\{x \in \mathbb{R}^{n} \mid \xi^{\prime} x \geq 0, \forall \xi \in F(\widehat{x})\right\}\right)\right] \\
& =\left\{0_{n}\right\} \cup \bigcup_{i=i}^{p}\left(\partial f_{i}(\widehat{x})\right)^{-},
\end{aligned}
$$

which amounts to saying that MOQ holds at $\widehat{x}$.

Obviously, the unique objective qualification in the above list, MOQ, is independent of the remaining data qualifications. Notice that, under the SSCQ, if $\psi$ is finite-valued, by [18, Proposition 1.3.3],

$$
\begin{aligned}
\operatorname{int} S & =\left\{x \in \mathbb{R}^{n}: \psi(x)<0\right\}, \\
\operatorname{bd} S & =\left\{x \in \mathbb{R}^{n}: \psi(x)=0\right\}, \\
\bar{S} & =\left\{x \in \mathbb{R}^{n}: \psi(x) \leq 0\right\} .
\end{aligned}
$$

In the particular case that $(P)$ is continuous and the SCQ holds then, by [14, Theorem 7.9], $\widehat{x} \in \operatorname{bd} S$ if and only if $G_{*}(\widehat{x}) \neq\left\{0_{n}\right\}$, which entails $G(\widehat{x}) \neq \emptyset$.

We illustrate the above data qualifications with a simple example showing that the PLVCQ is not implied by the remaining data qualifications.

Example 1 Consider the linear MOSIP problem in one variable

$$
\begin{aligned}
(P) \text { minimize } & f(x)=(-2 x,-x) \\
\text { subject to } & g_{0}(x)=2 x, \\
& g_{2 k+1}(x)=x-\frac{1}{k+1}, k=0,1,2, \ldots, \\
& g_{2 k}(x)=3 x-\frac{1}{k}, k=1,2, \ldots
\end{aligned}
$$

We observe that -1 is strong Slater point, $S=-\mathbb{R}_{+}, C(S, \widehat{x})=-\mathbb{R}_{+}$, $N(S, \widehat{x})=\mathbb{R}_{+}, T(\widehat{x})=\{0\}, G(\widehat{x})=\{2\}, G^{0}(\widehat{x})=-\mathbb{R}_{+}, G^{-}(\widehat{x})=-\mathbb{R}_{++}$, $G_{*}(\widehat{x})=\mathbb{R}_{+}, F(\widehat{x})=[-2,-1]$,

$$
\psi(x)=\left\{\begin{array}{l}
x, \text { if } x<0, \\
3 x, \text { else }
\end{array} \psi^{\prime}(\widehat{x} ; d)=\left\{\begin{array}{l}
-1, \text { if } d<0 \\
0, \text { if } d=0 \\
3, \text { else }
\end{array}\right.\right.
$$

and $\partial \psi(\widehat{x})=[1,3]$. Moreover, $F^{0}(\widehat{x}) \cap G^{0}(\widehat{x})=\mathbb{R}_{+} \cap\left(-\mathbb{R}_{+}\right)=\{0\}$, $C\left(Q^{1}(\widehat{x}), \widehat{x}\right) \cap C\left(Q^{2}(\widehat{x}), \widehat{x}\right)=\{0\},\left(\partial f_{1}(\widehat{x}) \cup \partial f_{2}(\widehat{x})\right)^{-}=\{-2,-1\}^{-}=$ $\mathbb{R}_{++}$, and $\sup _{t \in T_{\varepsilon}(\widehat{x})} g_{t}^{\prime}(\widehat{x})=\sup \{1,2,3\}=3$ independently of $\varepsilon>0$. Thus, SCQ, SSCQ, PMFCQ, LFMCQ, COCQ, MFCQ, KTCQ, CCCQ, ACQ, WADQ, EADQ, and MOQ hold at $\widehat{x}$ while PLVCQ fails at that point. 
Theorem 1 (Connections between data qualifications) Let $\widehat{x} \in S$. The implications of Diagram 1, where the local conditions are referred to $\widehat{x}$ and the label [1] ([2], [3], resp.) besides an arrow stands for 'the implication holds under the assumption that $(P)$ is continuous $(G(\widehat{x}) \neq \emptyset, p=1$, resp.)', hold true.

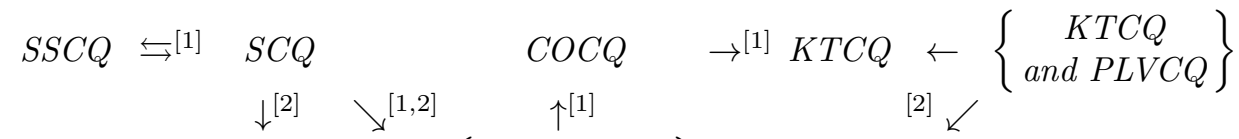

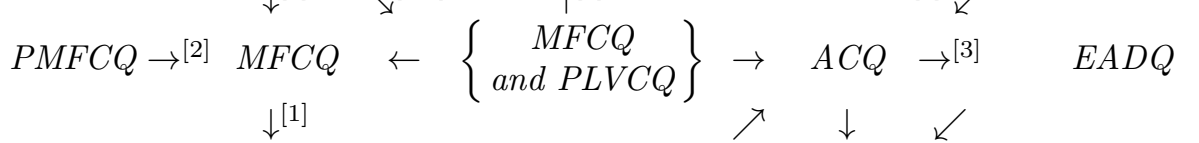

$$
\begin{aligned}
& L F M C Q \leftrightarrow\left\{\begin{array}{c}
A C Q \\
\text { and } C C C Q
\end{array}\right\} \quad W A D Q
\end{aligned}
$$

Diagram 1

Proof We first examine the case when $G(\widehat{x})=\emptyset$ regarding those implications involving the qualification conditions related with $G^{-}(\widehat{x})$ and $G^{0}(\widehat{x})$, that is, MFCQ, ACQ, WADQ, and EADQ. When [2] (i.e., the negation of $G(\widehat{x})=\emptyset)$ is required, there is nothing to be proved. On the one hand, since MFCQ fails, [MFCQ $\Longrightarrow$ LFMCQ] holds. On the other hand, since MFCQ, PLVCQ, ACQ, WADQ, and EADQ fail simultaneously, all implications of Diagram 1 involving ACQ, WADQ, and EADQ also hold. So, we can assume without loss of generality that $G(\widehat{x}) \neq \emptyset$.

$[\mathrm{SCQ} \Longrightarrow \mathrm{SSCQ}]$ : It is a straightforward consequence of the continuity assumption on $(P)$.

$[\mathrm{PMFCQ} \Longrightarrow \mathrm{MFCQ}]:$ Let $\varepsilon>0$ be such that $\sup \left\{\xi^{\prime} x_{*} \mid \xi \in \bigcup_{t \in T_{\varepsilon}(\widehat{x})} \partial g_{t}(\widehat{x})\right\}<$

0. Since $G(\widehat{x}) \subset \bigcup_{t \in T_{\varepsilon}(\widehat{x})} \partial g_{t}(\widehat{x}), \xi^{\prime} x_{*}<0$ for all $\xi \in G(\widehat{x})$, i.e., $x_{*} \in G^{-}(\widehat{x})$.

[SCQ $\Longrightarrow$ MFCQ]: If $x_{0}$ is a Slater point, $t \in T(\widehat{x})$ and $\xi_{t} \in \partial g_{t}(\widehat{x})$, then $\xi_{t}^{\prime}\left(x_{0}-\widehat{x}\right) \leq g_{t}\left(x_{0}\right)-g_{t}(\widehat{x})<0$, so that MFCQ holds at $\widehat{x}$ (actually at any point of $S)$.

[SCQ $\Longrightarrow \mathrm{PLVCQ}]:$ It is [18, Pshenichnyi-Levin-Valadier theorem, p. 267] (the continuity assumption is essential).

$[\mathrm{MFCQ} \Longrightarrow$ LFMCQ]: It is [13, Theorem 14(iii)].

$[\mathrm{LFMCQ} \Longrightarrow \mathrm{ACQ}]$ : By the assumption and the closedness of $C(S, \widehat{x})$,

$$
G^{0}(\widehat{x})=G_{*}^{0}(\widehat{x})=N^{0}(S, \widehat{x})=C^{00}(S, \bar{x})=\overline{C(S, \widehat{x})}=C(S, \widehat{x}) .
$$

[LFMCQ $\Longrightarrow \mathrm{CCCQ}$ ]: It follows from the closedness of $N(S, \widehat{x})$.

[(ACQ $\wedge \mathrm{CCCQ}) \Longrightarrow$ LFMCQ]: Taking negative polar cones in both sides of the inclusion $G_{*}^{0}(\widehat{x})=G^{0}(\widehat{x}) \subseteq C(S, \widehat{x})$, and recalling the closedness of 
$G_{*}(\widehat{x})$, one has

$$
N(S, \widehat{x})=C^{0}(S, \widehat{x}) \subseteq G_{*}^{00}(\widehat{x})=G_{*}(\widehat{x}),
$$

while the reverse inclusion holds by (4).

$[(\mathrm{KTCQ} \wedge \mathrm{PLVCQ}) \Longrightarrow \mathrm{ACQ}]$ : According to [28, Theorem 23.2],

$$
\begin{aligned}
(\partial \psi(\widehat{x}))^{0} & =\left\{d \in \mathbb{R}^{n} \mid \psi^{\prime}(\widehat{x} ; d) \geq \xi^{\prime} d \Longrightarrow \xi^{\prime} d \leq 0\right\} \\
& =\left\{d \in \mathbb{R}^{n} \mid \psi^{\prime}(\widehat{x} ; d) \leq 0\right\},
\end{aligned}
$$

which, together with $G(\widehat{x}) \neq \emptyset$, PLVCQ and KTCQ, yields

$$
\begin{gathered}
G^{0}(\widehat{x})=(\operatorname{conv}(G(\widehat{x})))^{0} \subseteq(\partial \psi(\widehat{x}))^{0}=\left\{d \in \mathbb{R}^{n} \mid \psi^{\prime}(\widehat{x} ; d) \leq 0\right\} \subseteq C(S, \widehat{x}) . \\
{[(\mathrm{MFCQ} \wedge \mathrm{PLVCQ}) \Longrightarrow \mathrm{COCQ}]: \text { Let } d \in G^{-}(\widehat{x}) . \text { Since } G^{-}(\widehat{x})=(\operatorname{conv}(G(\widehat{x})))^{-},}
\end{gathered}
$$

the PLVCQ leads to $d \in(\partial \psi(\widehat{x}))^{-}$. The continuity assumption [1] guarantees that $\widehat{x} \in \operatorname{int} \operatorname{dom} \psi=\mathbb{R}^{n}$, so that $\partial \psi(\widehat{x})$ is compact. Hence, by [28, Theorem 23.4],

$$
\psi^{\prime}(\widehat{x} ; d)=\max \left\{u^{\prime} d \mid u \in \partial \psi(\widehat{x})\right\}<0 .
$$

$[(\mathrm{MFCQ} \wedge \mathrm{PLVCQ}) \Longrightarrow \mathrm{ACQ}]:$ By the latter proof, we can take $d \in G^{-}(\widehat{x})$ such that $\psi^{\prime}(\widehat{x} ; d)<0$. Then, there exists a scalar $\delta>0$ such that $\psi(\widehat{x}+$ $\beta d)<\psi(\widehat{x}) \leq 0$, for all $\beta \in(0, \delta]$. Therefore, we have $\widehat{x}+\beta d \in S$ for all $\beta \in(0, \delta]$, which implies $d \in D(S, \widehat{x})$. We have thus proved the inclusion $G^{-}(\widehat{x}) \subseteq D(S, \widehat{x})$. Hence, we get

$$
G^{0}(\widehat{x})=\overline{G^{-}(\widehat{x})} \subseteq \overline{D(S, \widehat{x})}=C(S, \widehat{x}) .
$$

$[\mathrm{COCQ} \Longrightarrow \mathrm{KTCQ}]:$ Since $\psi^{\prime}(\widehat{x} ; \widehat{d})<0$, by the same argument as in the proof of $[(\mathrm{MFCQ} \wedge \mathrm{PLVCQ}) \Longrightarrow \mathrm{ACQ}]$, we obtain a $\delta>0$ such that $\widehat{x}+\delta \widehat{d} \in S$. Thus, recalling the definition of subdifferential, for each $t \in T(\widehat{x})$ and $\xi \in \partial g_{t}(\widehat{x})$, we have

$$
\xi^{\prime}(\delta \widehat{d})=\xi^{\prime}(\widehat{x}+\delta \widehat{d}-\widehat{x}) \leq g_{t}(\widehat{x}+\delta \widehat{d})-g_{t}(\widehat{x})=g_{t}(\widehat{x}+\delta \widehat{d}) \leq 0 .
$$

This means that $\left\{d \in \mathbb{R}^{n} \mid \psi^{\prime}(\widehat{x} ; d)<0\right\} \subseteq G^{0}(\widehat{x})$. This, together with the continuity of $\psi^{\prime}(\widehat{x} ;$.$) , implies that$

$$
\left\{d \in \mathbb{R}^{n} \mid \psi^{\prime}(\widehat{x} ; d) \leq 0\right\}=\overline{\left\{d \in \mathbb{R}^{n} \mid \psi^{\prime}(\widehat{x} ; d)<0\right\}} \subseteq \overline{G^{0}(\widehat{x})}=G^{0}(\widehat{x}) .
$$

The conclusion is immediate from the last inclusion and the definition of KTCQ.

$[\mathrm{ACQ} \Longrightarrow \mathrm{EADQ}]$ When $p=1$, the ACQ implies that

$$
F^{0}(\widehat{x}) \cap G^{0}(\widehat{x}) \subseteq G^{0}(\widehat{x}) \subseteq C(S, \widehat{x})=C\left(Q^{1}(\widehat{x}), \widehat{x}\right) .
$$


$[\mathrm{EADQ} \Longrightarrow \mathrm{WADQ}]$ In fact,

$$
F^{-}(\widehat{x}) \cap G^{0}(\widehat{x}) \subseteq F^{0}(\widehat{x}) \cap G^{0}(\widehat{x}) \subseteq \bigcap_{i=1}^{p} C\left(Q^{i}(\widehat{x}), \widehat{x}\right) \subseteq C(S, \widehat{x}) .
$$

The proof is complete.

Remark 1 Notice that assumption [1] can be relaxed in some cases. For instance, in the proofs of [(MFCQ $\wedge$ PLVCQ $) \Longrightarrow \mathrm{COCQ}],[(\mathrm{MFCQ} \wedge$ $\mathrm{PLVCQ}) \Longrightarrow \mathrm{ACQ}]$, and [COCQ $\Longrightarrow \mathrm{KTCQ}]$ we have used the compactness of $\partial \psi(\widehat{x})$, which follows from [1], but also from $\widehat{x} \in \operatorname{int} S($ as $S \subset \operatorname{dom} \psi)$.

The next, where we do not specify the objective function $f$, shows the necessity of the additional hypothesis [2] in the four implications of Diagram 1 where it is assumed.

Example 2 Take $n=1, T=[1,2], \widehat{x}=0$, and

$$
g_{t}(x)= \begin{cases}-\sqrt{2 t x-x^{2}}, & \text { if } x \in[0,2 t], \\ +\infty, & \text { otherwise }\end{cases}
$$

The function $(t, x) \longmapsto g_{t}(x)$ is continuous on $T \times S=[1,2] \times[0,2]$ but not on $T \times \mathbb{R}^{n}$, so that [1] fails. One has $T(\widehat{x})=T, \psi=g_{1}, C(S, \widehat{x})=\mathbb{R}_{+}, \psi^{\prime}(\widehat{x} ; d)=$ $-\infty$ for all $d>0, \partial g_{t}(\widehat{x})=\emptyset$ for all $t \in T, G(\widehat{x})=\emptyset$ (i.e., [2] fails despite of $\widehat{x} \in \operatorname{bd} S$ ), and $G_{*}(\widehat{x})=\{0\}$. Moreover, 1 is strong Slater point (with slack 1) and, taking an arbitrary $x_{*} \in \mathbb{R}^{n}$, we get $\left\{\xi^{\prime} x_{*} \mid \xi \in \bigcup_{t \in T_{\varepsilon}(\widehat{x})} \partial g_{t}(\widehat{x})\right\}=\emptyset$ for all $\varepsilon>0$, so that

$$
\inf _{\varepsilon>0} \sup \left\{\xi^{\prime} x_{*} \mid \xi \in \bigcup_{t \in T_{\varepsilon}(\widehat{x})} \partial g_{t}(\widehat{x})\right\}=-\infty<0 .
$$

Thus, SSCQ, SCQ, COCQ, KTCQ, PMFCQ, and PLVCQ hold while MFCQ, LFMCQ and ACQ fail. Then, [PMFCQ $\Longrightarrow$ MFCQ], [SCQ $\Longrightarrow$ MFCQ], $[\mathrm{SCQ} \Longrightarrow(\mathrm{MFCQ} \wedge \mathrm{PLVCQ})]$, and $[(\mathrm{KTCQ} \wedge \mathrm{PLVCQ}) \Longrightarrow \mathrm{ACQ}]$ fail.

Theorem 1 shows roughly speaking, first, that CCCQ and PLVCQ play subsidiary roles with respect to other CQs, second, that SSCQ is the strongest data qualification among those that are included in Diagram 1 and, third, that LFMCQ, KTCQ and WADQ are the weakest.

Example 3 Consider the MOSIP problem in $\mathbb{R}^{2}$

$$
\begin{array}{ll}
(P) \text { minimize } & f(x)=\left(-x_{1},-x_{1}\right) \\
\text { subject to } & g_{t}(x) \leq 0, t \in T:=\mathbb{N} \cup\{0\},
\end{array}
$$


where $f_{1}(x)=f_{2}(x):=-x_{1}$, and $g_{t}(x):=\sup \left\{x^{\prime} y \mid y \in X_{t}\right\}$ is the support function of the compact convex set $X_{t}:=\left\{x \in \mathbb{R}_{+}^{2} \mid x_{1}^{2}+x_{2}^{2}-2(1+t) x_{2} \leq 0\right\}$, so that $g_{t} \in \Gamma\left(\mathbb{R}^{n}\right)$ for all $t \in T$. Since $\left\{X_{t}\right\}_{t=1}^{\infty}$ is an expansive sequence of sets, $\left\{g_{t}\right\}_{t=1}^{\infty}$ is a non-decreasing sequence of nonnegative functions. Moreover, given $t \in T$, by a well-known property of the support functions, $\partial g_{t}(\widehat{x})=X_{t}$, while elementary calculus yields the explicit expression

$$
g_{t}(x)= \begin{cases}2(1+t) \max \left\{x_{2}, 0\right\} & , \text { if } x_{1} \leq 0, \\ \|x\|+(1+t) x_{2}, & \text { else }\end{cases}
$$

for the constraint functions. Thus, $\psi(x)=0$ when $x \in-\mathbb{R}_{+}^{2}$ and $\psi(x)=$ $+\infty$ otherwise, so that $S=-\mathbb{R}_{+}^{2}$.

We now take $\widehat{x}=0_{2}$. Then, $\psi^{\prime}(\widehat{x} ; d)=0$, if $d \in-\mathbb{R}_{+}^{2}$, and $\psi^{\prime}(\widehat{x} ; d)=+\infty$, otherwise while $\partial \psi(\widehat{x})=\mathbb{R}_{+}^{2}$. Moreover, $C(S, \widehat{x})=-\mathbb{R}_{+}^{2}, N(S, \widehat{x})=\mathbb{R}_{+}^{2}$, $T(\widehat{x})=T$,

$$
G_{*}(\widehat{x})=G(\widehat{x})=\left\{x \in \mathbb{R}^{2} \mid x_{1} \geq 0, x_{2}>0\right\} \cup\left\{0_{2}\right\},
$$

$G^{0}(\widehat{x})=-\mathbb{R}_{+}^{2}, G^{-}(\widehat{x})=\emptyset, F(\widehat{x})=\{(-1,0)\}, F^{0}(\widehat{x}) \cap G^{0}(\widehat{x})=\{0\} \times\left(-\mathbb{R}_{+}\right)$, $C\left(Q^{1}(\widehat{x}), \widehat{x}\right) \cap C\left(Q^{2}(\widehat{x}), \widehat{x}\right)=\{0\} \times\left(-\mathbb{R}_{+}\right), \bigcup_{i=i}^{p}\left(\partial f_{i}(\widehat{x})\right)^{-}=\mathbb{R}_{++} \times \mathbb{R}$, and $\bigcup_{t \in T_{\varepsilon}(\widehat{x})} \partial g_{t}(\widehat{x})=\left(\mathbb{R} \times \mathbb{R}_{++}\right) \cup\left\{0_{2}\right\}$ for all $\varepsilon>0$. Therefore, KTCQ, ACQ, WADQ, and EADQ hold at $\widehat{x}$ while the remaining data qualifications in Theorem 1 fail (as well as $M O Q$ ). Observe also that int $S \neq \emptyset=$ $\left\{x \in \mathbb{R}^{n}: \psi(x)<0\right\}$, so that SSCQ is essential for the validity of ([6).

\section{Optimality conditions for weak efficiency}

Recall that we consider a problem $(P)$ as in (1) with convex and finitevalued objective functions $f_{i}, i \in I$, and constraint functions $g_{t} \in \Gamma\left(\mathbb{R}^{n}\right)$, $t \in T$.

We say that the weak KKT condition holds at $\widehat{x} \in S$ when there exist $\alpha_{i} \geq 0$ for $i \in I$ with $\sum_{i=1}^{p} \alpha_{i}=1$, and $\beta_{t} \geq 0$ for $t \in T(\widehat{x})$, with $\beta_{t} \neq 0$ for finitely many indexes, such that

$$
0_{n} \in \sum_{i=1}^{p} \alpha_{i} \partial f_{i}(\widehat{x})+\sum_{t \in T(\widehat{x})} \beta_{t} \partial g_{t}(\widehat{x})
$$

In geometric terms, the weak KKT condition holds at $\widehat{x} \in S$ if and only if $0_{n} \in F_{*}(\widehat{x})+G_{*}(\widehat{x})$.

Theorem 2 (Weak KKT necessary condition under WACQ and CCCQ) Let $\widehat{x}$ be a weak efficient solution of problem $(P)$. Then:

(i) If WACQ holds at $\widehat{x}$, one has

$$
0_{n} \in F_{*}(\widehat{x})+\overline{G_{*}(\widehat{x})}
$$


(ii) If, in addition, CCCQ holds at $\widehat{x}$, then $(P)$ satisfies the weak $K K T$ condition at $\widehat{x}$.

Proof (i) We first claim that

$$
\max _{i \in I} f_{i}^{\prime}(\widehat{x} ; d) \geq 0, \quad \forall d \in D(S, \widehat{x}) .
$$

On the contrary, suppose that there exists a $d \in D(S, \widehat{x})$ such that $f_{i}^{\prime}(\widehat{x} ; d)<$ 0 for all $i \in I$. Thus, there exist positive scalars $\delta, \delta_{1},,,,, \delta_{p}$ such that

$$
\left\{\begin{array}{l}
\widehat{x}+\varepsilon d \in S, \quad \forall \varepsilon \in(0, \delta), \\
f_{i}(\widehat{x}+\varepsilon d)-f_{i}(\widehat{x})<0, \forall \varepsilon \in\left(0, \delta_{i}\right) .
\end{array}\right.
$$

Take $\widehat{\delta}:=\min \left\{\delta, \delta_{1},,,,, \delta_{p}\right\}$. From (10), for each $\varepsilon \in(0, \widehat{\delta})$ we get $f(\widehat{x}+\varepsilon d)<$ $f(\widehat{x})$ and $\widehat{x}+\varepsilon d \in S$, which contradicts the weak efficiency of $\widehat{x}$. Thus, (9) is true.

We now show that (9) also holds for $d \in C(S, \widehat{x})=\overline{D(S, \widehat{x})}$. Indeed, if $d \in \overline{D(S, \widehat{x})}$, there exists a sequence $\left\{d_{k}\right\}_{k=1}^{\infty}$ in $D(S, \widehat{x})$ converging to $d$. For each $i \in I$, since $f_{i}$ is a finite convex function, its directional derivative function $f_{i}^{\prime}(\widehat{x} ; \cdot)$ at $\widehat{x}$ is finite sublinear [18, Proposition 1.1.2] and, so, convex and continuous. Thus, $\varphi(\cdot):=\max _{i \in I} f_{i}^{\prime}(\widehat{x} ; \cdot)$ is a convex continuous function too. From (9) and the continuity of $\varphi$ we deduce that $\varphi(d)=\lim _{k \rightarrow \infty} \varphi\left(d_{k}\right) \geq 0$, so that $\varphi(d) \geq 0$ for all $d \in C(S, \widehat{x})$. From this inequality and the ACQ at $\widehat{x}$, we obtain that

$$
\varphi(d) \geq 0, \forall d \in F^{-}(\hat{x}) \cap G^{0}(\widehat{x}) .
$$

We claim that $F^{-}(\hat{x}) \cap G^{0}(\hat{x})=\emptyset$. Otherwise, if $\hat{d} \in F^{-}(\hat{x}) \cap G^{0}(\hat{x})$, then $f_{i}^{\prime}(\hat{x} ; \hat{d})<0$ for all $i \in I$ (by definition of $F^{-}(\hat{x})$ ), so that $\varphi(\hat{d})<0$, which contradicts (11). Thus our claim is proved. Since $F^{-}(\hat{x})=F_{*}^{-}(\hat{x})$ and $G^{0}(\hat{x})=\left({\overline{G_{*}(\hat{x})}}^{0}\right.$, then $F_{*}^{-}(\hat{x}) \cap\left({\overline{G_{*}(\hat{x})}}^{0}=\emptyset\right.$. Hence there is no vector $v \in \mathbb{R}^{n}$ satisfying

$$
\left\{\begin{array}{l}
v^{\prime} y<0, \quad \forall y \in F_{*}(\hat{x}), \\
v^{\prime} y \geq 0, \quad \forall y \in\left(-\overline{G_{*}(\hat{x})}\right) .
\end{array}\right.
$$

Since $F_{*}(\hat{x})$ is a non-empty convex set and $\left(-\overline{G_{*}(\hat{x})}\right)$ is a closed convex cone, by the strong separation theorem (see, e.g., [28, Corollary 11.4.1] we get $F_{*}(\hat{x}) \cap\left(-\overline{G_{*}(\hat{x})}\right) \neq \emptyset$. This means that

$$
\left.0_{n} \in F_{*}(\hat{x}) \cap \overline{G_{*}(\hat{x})}\right) .
$$

(ii) Under the closedness assumption, $0_{n} \in F_{*}(\widehat{x})+G_{*}(\widehat{x})$ and the conclusion follows. 
Theorem 3 (Weak KKT sufficient condition) If the weak KKT condition holds at $\widehat{x} \in S$, then, $\widehat{x}$ is a weak efficient solution of $(P)$.

Proof Let $\alpha_{i} \geq 0, i \in I$, with $\sum_{i=1}^{p} \alpha_{i}=1$, and some $\beta_{t} \geq 0, t \in T(\widehat{x})$, with $\beta_{t} \neq 0$ for finitely many indexes.Due to (7), we can find some $\xi_{i} \in \partial f_{i}(\widehat{x})$ and $\zeta_{t} \in \partial g_{t}(\widehat{x})$ for $(i, t) \in I \times T(\widehat{x})$ such that

$$
\sum_{i=1}^{p} \alpha_{i} \xi_{i}+\sum_{t \in T^{*}} \beta_{t} \zeta_{t}=0_{n}
$$

where $T^{*}:=\left\{t \in T(\widehat{x}) \mid \beta_{t} \neq 0\right\}$. Suppose on the contrary that $\widehat{x}$ is not a weak efficient solution for $(P)$. Then there exists a feasible point $x_{*}$ for $(P)$ such that $f_{i}\left(x_{*}\right)<f_{i}(\widehat{x})$ for all $i \in I$. Thus, $\xi_{i}^{\prime}\left(x_{*}-\widehat{x}\right)<0$ for all $i \in I$. Due to the last inequality, it follows from (12) that

$$
\sum_{t \in T^{*}} \beta_{t} \zeta_{t}^{\prime}\left(x_{*}-\widehat{x}\right)=-\sum_{i=1}^{p} \alpha_{i} \xi_{i}^{\prime}\left(x_{*}-\widehat{x}\right)>0 .
$$

On the other hand, since $T^{*} \subseteq T(\widehat{x})$, we obtain that

$$
\sum_{t \in T^{*}} \beta_{t} \zeta_{t}^{\prime}\left(x_{*}-\widehat{x}\right) \leq 0
$$

This contradicts (13).

Combining Theorems 1, 2 and 3 we get the following characterization of the weak efficient solution for (possibly non-continuous) convex MOSIP problems.

Corollary 1 (Characterization under LFMCQ via weak KKT condition) Suppose that $(P)$ satisfies $L F M C Q$ at $\widehat{x} \in S$. Then, $\widehat{x}$ is a weak efficient solution for $(P)$ if and only if $(P)$ satisfies the weak KKT condition at $\widehat{x}$.

A similar result was proved, for continuous convex MOSIP problems, in [13, Theorem 27] under the MFCQ and in [13, Theorem 29] under the KTCQ and the CCCQ, and the assumption that $0_{n} \notin F_{*}(\widehat{x})$.

We now exploit the gap function associated with $(P)$ to characterize its weak efficient solutions.

Theorem 4 (Characterization under LFMCQ via gap function) Let $\widehat{x} \in S$. The following statements hold:

(i) If $\vartheta(\widehat{x}, \xi, \lambda)=0$ for some $\xi:=\left(\xi_{1}, \ldots, \xi_{p}\right) \in \prod_{i=1}^{p}\left(\partial f_{i}(\widehat{x})\right)$ and $\lambda \geq 0_{p}$, then $\widehat{x}$ is a weak efficient solution for $(P)$.

(ii) If $\widehat{x}$ is a weak efficient solution for $(P)$ where the LFMCQ holds, then there exist $\xi \in \prod_{i=1}^{p} \partial f_{i}(\widehat{x})$ and $\lambda \geq 0_{p}$ such that $\vartheta(\widehat{x}, \xi, \lambda)=0$. 
Proof (i) Assume that $\vartheta(\widehat{x}, \xi, \lambda)=0$, while $\widehat{x}$ is not a weak efficient solution for $(P)$. Then, there exists $x^{*} \in S$ such that

$$
f_{i}\left(x^{*}\right)<f_{i}(\widehat{x}), \forall i \in I .
$$

Then, by definition of subgradient,

$$
\xi_{i}^{\prime}\left(x^{*}-\widehat{x}\right)<0, \forall i \in I .
$$

Due to the latter inequalities and the assumption that $\lambda \geq 0_{p}$, we have $\sum_{i=1}^{p} \lambda_{i} \xi_{i}^{\prime}\left(\widehat{x}-x^{*}\right)>0$. Hence, $\vartheta(\widehat{x}, \xi, \lambda)>0$, which contradicts the assumption.

(ii) According to Corollary 1, there exist $\lambda:=\left(\lambda_{1},,,,, \lambda_{p}\right) \geq 0_{p}$ with $\sum_{i=1}^{p} \lambda_{i}=1$, a finite set $\left\{t_{1},,,,, t_{q}\right\} \subseteq T(\widehat{x})$, with corresponding nonnegative scalars $\mu_{t_{1}},,,,, \mu_{t_{q}}$, and subgradients $\xi_{i} \in \partial f_{i}(\widehat{x})$ for $i \in I$, and $\zeta_{t_{m}} \in \partial g_{t_{m}}(\widehat{x})$ for $m=1,,,,, q$, such that

$$
\sum_{i=1}^{p} \lambda_{i} \xi_{i}+\sum_{m=1}^{q} \mu_{t_{m}} \zeta_{t_{m}}=0_{n}
$$

Take an arbitrary $y \in S$ and $m \in\{1, \ldots, q\}$. Since $g_{t_{m}}(y) \leq 0=g_{t_{m}}(\widehat{x})$, $\zeta_{t_{m}}^{\prime}(y-\widehat{x}) \leq 0$. This and (14) imply that:

$$
\sum_{i=1}^{p} \lambda_{i} \xi_{i}^{\prime}(y-\widehat{x})=-\sum_{m=1}^{q} \mu_{t_{m}} \zeta_{t_{m}}^{\prime}(y-\widehat{x}) \geq 0 .
$$

Therefore, $\sum_{i=1}^{p} \lambda_{i} \xi_{i}^{\prime}(\widehat{x}-y) \leq 0$ for all $y \in S$. From this and $\sum_{i=1}^{p} \lambda_{i} \xi_{i}^{\prime}(\widehat{x}-$ $\widehat{x})=0$, we conclude that

$$
\vartheta(x, \xi, \lambda)=\sup _{y \in S}\left\{\sum_{i=1}^{p} \lambda_{i} \xi_{i}^{\prime}(x-y)\right\}=0 .
$$

Finally in this section, we consider $(P)$ as a particular instance of the general vector optimization problem studied in [8, just taking as decision space $X=\mathbb{R}^{n}$, as objective space $Y=\mathbb{R}^{p}$, and as constraint space $Z$ some linear subspace of $\mathbb{R}^{T}$ such that $Z^{\bullet}:=Z \cup\left\{+\infty_{Z}\right\}$, where $+\infty_{Z}$ denotes a greatest element, contains $\left\{g(x) \mid x \in \mathbb{R}^{n}\right\}$, where $g(x):=\left(g_{t}(x)\right)_{t \in T}$ for all $x \in \mathbb{R}^{n}$, with the convention that $g(x)=+\infty_{Z}$ when $g_{t}(x)=+\infty$ for at least one $t \in T$. The domain of $g$ is

$$
\operatorname{dom} g:=\left\{x \in \mathbb{R}^{n} \mid g(x) \neq+\infty_{Z}\right\}=\bigcap_{t \in T} \operatorname{dom} g_{t} \neq \emptyset .
$$

We assume that $Z$ is equipped with a locally convex topology finer than the one of the pointwise convergence, with positive cone $Z \cap \mathbb{R}_{+}^{T}$. We consider on the dual space of $Z, Z^{*}$, the ordering induced by the positive cone $Z \cap \mathbb{R}_{+}^{T}$, i.e., the positive cone in $Z^{*}$ is $Z_{+}^{*}:=\left\{z^{*} \in Z^{*} \mid\left\langle z^{*}, z\right\rangle \geq 0, \forall z \in Z \cap \mathbb{R}_{+}^{T}\right\}$. Given $z^{*} \in Z^{*}$, we define $\left(z^{*} \circ g\right)(x)=+\infty$ whenever $g(x)=+\infty_{Z}$. 
The conjugate of a vector function $h: \mathbb{R}^{n} \longrightarrow \mathbb{R}^{p} \cup\left\{+\infty_{\mathbb{R}^{p}}\right\}$, where $+\infty \mathbb{R}^{p}$ denotes an element greater than any other in $\mathbb{R}^{p}$, is the set-valued $\operatorname{map} h^{*}: \mathcal{M}_{p \times n} \rightrightarrows \mathbb{R}^{p} \cup\left\{+\infty_{\mathbb{R}^{p}}\right\}$ defined by

$$
h^{*}(M):=\operatorname{WSup}\left\{M x-h(x) \mid x \in \mathbb{R}^{n}\right\}, \forall M \in \mathcal{M}_{p \times n},
$$

where WSup $V$ represents the weak supremum of $V \subset \mathbb{R}^{p}$ in Tanino's sense 32]. Due to the assumptions on the data of $(P)$, the next lemma is a straightforward consequence of [8, Theorem 5.1].

Lemma 1 Let $\widehat{x} \in S$. Then the following statements are equivalent:

(i) The set

$$
\bigcup_{\left(z_{1}^{*}, \ldots, z_{p}^{*}\right) \in\left(Z_{+}^{*}\right)^{p}}\left\{(M, y) \in \mathcal{M}_{p \times n} \times \mathbb{R}^{p} \mid y \in\left(f+\left(z_{1}^{*}, \ldots, z_{p}^{*}\right) \circ g\right)^{*}(M)+\mathbb{R}_{+}^{p}\right\}
$$

is closed regarding $\left(0_{p \times n},-f(\widehat{x})\right)$.

(ii) $\widehat{x}$ is a weak efficient solution of $(P)$ if and only if there exist $z_{1}^{*}, \ldots, z_{p}^{*} \in$ $Z_{+}^{*}$ such that

$$
f(x)+\left(\left\langle z_{1}^{*}, g(x)\right\rangle, \ldots,\left\langle z_{p}^{*}, g(x)\right\rangle\right)-f(\widehat{x}) \notin-\mathbb{R}_{++}^{p}, \forall x \in \mathbb{R}^{n} .
$$

Observe that (16) holds whenever $\widehat{x}$ is a weak efficient solution for the ordinary multiobjective problem

$$
\text { minimize } f(x) \text { subject to }\left\langle z_{i}^{*}, g(x)\right\rangle \leq 0, i \in I \text {. }
$$

We now get from Lemma 1 characterizations of the weak efficient solutions involving continuous linear functionals on $Z$ instead of subgradients of the data at $\widehat{x}$ (as in the KKT-type theorems) for three particular types of convex MOSIP problems.

Theorem 5 (Characterization under SSCQ and SCQ via linear functionals) Let $\widehat{x} \in S$. Then the following statements hold:

(i) Assume that $T$ is countable and $0_{\mathbb{R}^{T}} \in$ sqri $E$, where

$$
E:=g\left(\bigcap_{t \in T} \operatorname{dom} g_{t}\right)+\mathbb{R}_{+}^{T}
$$

Then $\widehat{x}$ is a weak efficient solution of $(P)$ if and only if there exist multiplier vectors $\lambda^{1}, \ldots, \lambda^{p} \in \mathbb{R}_{+}^{T}$, all of them with finite support, such that there is no $x \in \mathbb{R}^{n}$ satisfying

$$
f_{i}(x)+\sum_{t \in T} \lambda_{t}^{i} g_{t}(x)<f(\widehat{x}), \forall i \in I .
$$

(ii) Assume that $T$ is a normal topological space space, the functions $(\cdot, x) \mapsto$ $g$. $(x)$ are continuous for all $x \in \mathbb{R}^{n}$, the supremum and infimum functions $\psi$ and $\iota$ (defined in (2) and (3), respectively) are real-valued, and SSCQ 
holds. Then $\widehat{x}$ is a weak efficient solution of $(P)$ if and only if there exist nonnegative bounded finitely additive measures $\mu_{1}, \ldots, \mu_{p}$ such that there is no $x \in \mathbb{R}^{n}$ satisfying

$$
f_{i}(x)+\int_{T} g_{t}(x) d \mu_{i}(t)<f(\widehat{x}), \forall i \in I .
$$

(iii) Assume that $(P)$ is continuous and either $0_{\mathcal{C}(T)} \in \mathrm{ri}\left[g\left(\mathbb{R}^{n}\right)+\left(Z \cap \mathbb{R}_{+}^{T}\right)\right]$ or the SCQ holds. Then $\widehat{x}$ is a weak efficient solution of $(P)$ if and only if there exist $p$ nonnegative regular Borel measures on $T, \mu_{1}, \ldots, \mu_{p}$, such that there is no $x \in \mathbb{R}^{n}$ satisfying

$$
f_{i}(x)+\int_{T} g_{t}(x) d \mu_{i}(t)<f(\widehat{x}), \forall i \in I .
$$

Proof According to [8, Lemma 3.8], any of the following CQs guarantees the closedness of the set in (15), and so the fulfillment of statement $(i)$ in Lemma 1:

Q1: There exists $x_{0} \in \mathbb{R}^{n}$ such that $g\left(x_{0}\right) \in-\operatorname{int}\left(Z \cap \mathbb{R}_{+}^{T}\right)$.

Q2: $Z$ is a Fréchet space and $0_{Z} \in \operatorname{sqri}\left(g\left(\bigcap_{t \in T} \operatorname{dom} g_{t}\right)+\left(Z \cap \mathbb{R}_{+}^{T}\right)\right)$.

So, we have just to show that at either Q1 or Q2 holds under the assumptions of $(i)$ - (iii), so that the conclusion follows from Lemma 1 .

(i) Take $Z=\mathbb{R}^{T}$ equipped with the product topology. Observe that the topological dual $Z^{*}$ of $Z$ is here the subspace of $\mathbb{R}^{T}$ formed by the functions with finite support. It can be realized that $Z$ is a Fréchet space if and only if $T$ is countable. Moreover, $y^{*} \circ f$ is continuous for all $y^{*} \in \mathbb{R}^{p}$, so that Q2 holds.

(ii) Since $-\infty<\iota(x) \leq g_{t}(x) \leq \psi(x)<+\infty$ for all $t \in T$ and $x \in \mathbb{R}^{n}$, $g: \mathbb{R}^{n} \rightarrow Z$, where $Z:=\{h \in \mathcal{C}(T) \mid h$ is bounded $\}$. Since $T$ is a normal space, its dual space $Z^{*}$ is formed by the bounded finitely additive measures on $T$ [9, Theorem IV.6.2]. Moreover, it is easy to see that int $\left(Z \cap \mathbb{R}_{+}^{T}\right)=$ $\left\{h \in Z: \inf _{T} h>0\right\}$. If $x_{0}$ is a strong Slater point with associated scalar $\varepsilon$, then $\inf _{T}\left[-g\left(x_{0}\right)\right] \geq \varepsilon>0$, so that $g\left(x_{0}\right) \in-\operatorname{int}\left(Z \cap \mathbb{R}_{+}^{T}\right)$ and Q1 holds. (iii) The continuity assumption implies that $g: \mathbb{R}^{n} \rightarrow \mathcal{C}(T)$. Take $Z=$ $\mathcal{C}(T)$ equipped with the supremum norm, whose topological dual is formed by the regular Borel measures on $T$. Under the SCQ, Q1 holds by the same argument as in (ii), while, taking into account that any Banach space is Fréchet and $\operatorname{dom} g_{t}=\mathbb{R}^{n}$ for all $t \in T$ in this case, $(Q 2)$ is equivalent here to $0_{Z} \in \operatorname{ri}\left[g\left(\mathbb{R}^{n}\right)+\left(Z \cap \mathbb{R}_{+}^{T}\right)\right]$.

We now revisit Examples 1 and 3 .

In Example 1, $(P)$ satisfies LFMCQ at $\widehat{x}=0$. Since $F_{*}(\widehat{x})+G_{*}(\widehat{x})=$ $[-2,-1]+\mathbb{R}_{+}=[-2,+\infty) \ni 0$, the weak KKT condition holds at $\widehat{x}=0$. Moreover, $\xi=(-2,-1)$ is fixed and

$$
\vartheta(x, \lambda)=-\left(2 \lambda_{1}+\lambda_{2}\right) \inf \{x-y \mid y \leq 0\}=-\left(2 \lambda_{1}+\lambda_{2}\right) x,
$$


so that $\vartheta(\widehat{x}, \lambda)=0$ for all $\lambda \in \Delta_{+}^{2}$. Concerning Theorem [5 notice that the index set $T$ (equipped with the metric induced by the absolute value) is countable, it is normal (as it is metric) and the functions $(\cdot, x) \mapsto g .(x)$ are continuous for all $x \in \mathbb{R}^{n}$ (as the topology on $T$ is the discrete one), but the condition $0_{\mathbb{R}^{T}} \in$ sqri $\left[g\left(\mathbb{R}^{n}\right)+\mathbb{R}_{+}^{T}\right]$ can hardly be checked (as the set $g\left(\mathbb{R}^{n}\right)+\mathbb{R}_{+}^{T}$ is here the sum of the cone of nonnegative sequences with the line in $\mathbb{R}^{T}$ which passes through the sequence $\left\{u_{k}\right\}_{k=0}^{\infty}$ such that $u_{0}=0$, $u_{2 k+1}=-\frac{1}{k+1}, k=0,1,2, \ldots$, and $u_{2 k}=-\frac{1}{k}, k=1,2, \ldots$, and is parallel to the sequence $\left\{v_{k}\right\}_{k=0}^{\infty}$ such that $v_{0}=2, v_{t}=1$ for $t$ even, and $v_{t}=3$ for $t$ odd). Observe that, in either case, the optimality condition in $(i)$ holds for $\lambda^{1} \in \mathbb{R}^{T}$ such that $\lambda_{0}^{1}=1$ and $\lambda_{t}^{1}=0$ for all $t \in \mathbb{N}$, and $\lambda^{2} \in \mathbb{R}^{T}$ arbitrary with finite , as $f_{1}+\lambda_{0}^{1} g_{0}$ is the null function on $\mathbb{R}$. Moreover, since $\psi$ and $\iota(x)=\min \{x-1,3 x-1\}$ are real-valued and SSCQ holds, (ii) applies, with $\mu_{1}$ being the atomic measure concentrating a unit mass at 0 and $\mu_{2}$ arbitrary as $f_{1}(x)+\int_{T} g_{t}(x) d \mu_{i}(t)=f_{1}(x)+g_{0}(x)=0$ for all $x \in \mathbb{R}$. So, we can assert that $\widehat{x}$ is a weak efficient solution for $(P)$ on the basis of Theorems 3 , 4 and 5 .

The situation is quite different at $\widehat{x}=0_{2}$ in Example3, where ACQ holds while CCCQ, LFMCQ, and the weak KKT condition fail. This means that no conclusion on the weak efficiency of $\widehat{x}$ can be obtained from Theorems 2 and 3. Concerning the gap function, $\xi=(-1,0,-1,0)$ is also fixed and

$$
\vartheta(x, \lambda)=\left(\lambda_{1}+\lambda_{2}\right) \sup \left\{y_{1}-x_{1} \mid y_{1} \leq 0\right\}=-\left(\lambda_{1}+\lambda_{2}\right) x_{1},
$$

so that we have again $\vartheta(\widehat{x}, \lambda)=0$ for all $\lambda \in \Delta_{+}^{2}$. Regarding Theorem 5] observe that $T$ is the same as in Example 1, but neither (ii) nor (iii) can be applied because SSCQ fails and $\psi$ is not real-valued, respectively, while checking the interiority condition in $(i)$ is again a hard task. So, only Theorem 4 allows to conclude easily that $\widehat{x}$ is a weak efficient solution. This example shows that we cannot replace LFMCQ by ACQ, KTCQ or WADQ in Corollary 1 .

\section{Optimality conditions for efficiency}

We say that the strong KKT condition holds at $\widehat{x} \in S$ when there exist $\alpha_{i}>0$ for $i \in I$ with $\sum_{i=1}^{p} \alpha_{i}=1$, and $\beta_{t} \geq 0$ for $t \in T(\widehat{x})$, with $\beta_{t} \neq 0$ for finitely many indexes, such that

$$
0_{n} \in \sum_{i=1}^{p} \alpha_{i} \partial f_{i}(\widehat{x})+\sum_{t \in T(\widehat{x})} \beta_{t} \partial g_{t}(\widehat{x})
$$

Denoting $\Delta_{++}^{p}:=\left\{\left(\alpha_{1}, \ldots, \alpha_{p}\right) \in \mathbb{R}_{++}^{p} \mid \sum_{i=1}^{p} \alpha_{i}=1\right\}$, 28, Theorem 6.9] yields

$$
\operatorname{ri} F_{*}(\widehat{x})=\bigcup_{\left(\alpha_{1}, \ldots, \alpha_{p}\right) \in \Delta_{++}^{p}} \sum_{i=1}^{p} \alpha_{i} \operatorname{ri} \partial f_{i}(\widehat{x}) \subseteq \bigcup_{\left(\alpha_{1}, \ldots, \alpha_{p}\right) \in \Delta_{++}^{p}} \sum_{i=1}^{p} \alpha_{i} \partial f_{i}(\widehat{x}),
$$


and the inclusion is an equation whenever $f_{i}$ is differentiable at $\widehat{x}$ for all $i \in I$. Thus, $0_{n} \in \operatorname{ri} F_{*}(\widehat{x})+G_{*}(\widehat{x})$ is a sufficient condition for the strong KKT condition at $\widehat{x}$ and it is also necessary when the objective functions are differentiable at $\widehat{x}$.

Theorem 6 (Strong KKT necessary condition under EADQ and MOQ) Let $\widehat{x}$ be an efficient solution of $(P)$. If the EADQ and the $M O Q$ hold at $\widehat{x}$, then $(P)$ satisfies the strong KKT condition at $\widehat{x}$.

Proof For the sake of simplicity, we replace $Q^{l}(\widehat{x})$ by $Q^{l}$ in this section. We can assume without loss of generality that $p \geq 2$. We present the proof in four steps.

Step 1. We claim that

$$
\left(\partial f_{l}(\widehat{x})\right)^{-} \cap D\left(Q^{l}, \widehat{x}\right)=\emptyset, \forall l \in I .
$$

On the contrary, suppose that for some $l \in I$ there is a vector $d$ such that

$$
d \in\left(\partial f_{l}(\widehat{x})\right)^{-} \cap D\left(Q^{l}, \widehat{x}\right) .
$$

By the definition of $D\left(Q^{l}, \widehat{x}\right)$, there exists a $\delta>0$ such that $\widehat{x}+\varepsilon d \in Q^{l}$ for each $\varepsilon \in(0, \delta)$. Thus, due to the definition of $Q^{l}$, we obtain that

$$
\left\{\begin{array}{l}
f_{i}(\widehat{x}+\varepsilon d) \leq f_{i}(\widehat{x}), \forall i \in I \backslash\{l\}, \forall \varepsilon \in(0, \delta), \\
\widehat{x}+\varepsilon d \in S, \quad \forall \varepsilon \in(0, \delta) .
\end{array}\right.
$$

On the other hand, (21) leads to $f_{l}^{\prime}(\widehat{x} ; d)<0$. This means that there exists a $\delta_{l}>0$ satisfying

$$
f_{l}(\widehat{x}+\varepsilon d)-f_{l}(\widehat{x})<0, \forall \varepsilon \in\left(0, \delta_{l}\right) .
$$

Taking $\widehat{\delta}:=\min \left\{\delta, \delta_{l}\right\}$ and $\varepsilon \in(0, \widehat{\delta})$, (23) and (22) contradict the efficiency of $\widehat{x}$. Therefore, our claim holds.

Step 2. Let $\widehat{d} \in C\left(Q^{l}, \widehat{x}\right)=\overline{D\left(Q^{l}, \widehat{x}\right)}$ for some $l \in I$. Then, there exists a sequence $\left\{d_{k}\right\}_{k=1}^{\infty}$ in $D\left(Q^{l}, \widehat{x}\right)$ converging to $\widehat{d}$. By (20) and the continuity of $f_{l}^{\prime}(\widehat{x} ;$.$) we get$

$$
f_{l}^{\prime}(\hat{x} ; \hat{d})=f_{l}^{\prime}\left(\hat{x} ; \lim _{n \rightarrow \infty} d_{k}\right)=\lim _{n \rightarrow \infty} f_{l}^{\prime}\left(\hat{x} ; \hat{d}_{k}\right) \geq 0,
$$

so that

$$
\left(\partial f_{l}(\widehat{x})\right)^{-} \cap C\left(Q^{l}, \widehat{x}\right)=\emptyset, \forall l \in I .
$$

Hence,

$$
\left(\bigcup_{i=1}^{p}\left(\partial f_{i}(\widehat{x})\right)^{-}\right) \cap\left(\bigcap_{i=1}^{p} C\left(Q^{i}, \widehat{x}\right)\right)=\emptyset .
$$

Step 3. We claim that

$$
0_{n} \in \operatorname{ri}\left(F_{*}(\widehat{x})\right)+G_{*}(\widehat{x}) .
$$


On the contrary, suppose that (25) does not hold. Then ri $\left(F_{*}(\widehat{x})\right) \cap(-$ $\left.G_{*}(\widehat{x})\right)=\emptyset$. Thus, by the proper separation theorem ([28, Theorem 11.3]) and noting that $\left(-G_{*}(\widehat{x})\right)$ is a convex cone, it follows that there is a hyperplane $H_{d}:=\left\{x \in \mathbb{R}^{n} \mid d^{\prime} x=0\right\}$ for some $d \in \mathbb{R}^{n} \backslash\{0\}$ separating $F_{*}(\widehat{x})$ and $\left(-G_{*}(\widehat{x})\right)$ properly. In other words, there exists a vector $d \in \mathbb{R}^{n}$ satisfying $0_{n} \neq d \in\left(F_{*}(\widehat{x})\right)^{0} \cap\left(G_{*}(\widehat{x})\right)^{0}=F^{0}(\widehat{x}) \cap G^{0}(\widehat{x})$. Thus, owning to EADQ and MOQ we conclude that

$$
d \in\left(\bigcup_{i=1}^{p}\left(\partial f_{i}(\widehat{x})\right)^{-}\right) \cap\left(\bigcap_{i=1}^{p} C\left(Q^{i}, \widehat{x}\right)\right),
$$

which contradicts (24).

Step 4. The result is immediate from (25) and the fact that (see, 28, Theorem 6.9])

$$
\operatorname{ri}\left(F_{*}(\widehat{x})\right) \subseteq\left\{\sum_{i=1}^{p} \alpha_{i} \xi_{i} \mid \xi_{i} \in \partial f_{i}(\widehat{x}), \alpha_{i}>0, \sum_{i=1}^{p} \alpha_{i}=1\right\} .
$$

The proof of the following theorem is exactly the same as the one of Theorem 3, and so has been omitted.

Theorem 7 (Strong KKT sufficient condition) If the strong KKT condition hods at $\widehat{x} \in S$, then, $\widehat{x}$ is an efficient solution of $(P)$.

We now characterize the efficiency through the gap function.

Theorem 8 (Characterization under EADQ and MOQ via gap function) Let $\widehat{x} \in S$. The following statements hold true:

(i) If $\vartheta(\widehat{x}, \xi, \lambda)=0$ for some $\xi:=\left(\xi_{1}, \ldots, \xi_{p}\right) \in \prod_{i=1}^{p}\left(\partial f_{i}(\widehat{x})\right)$ and $\lambda>0_{p}$, then $\widehat{x}$ is an efficient solution for $(P)$.

(ii) If $\widehat{x}$ is an efficient solution for $(P)$ where the EADQ and the MOQ hold, then there exist $\xi \in \prod_{i=1}^{p} \partial f_{i}(\widehat{x})$ and $\lambda>0_{p}$ such that $\vartheta(\widehat{x}, \xi, \lambda)=0$.

Proof $(i)$ Assume that $\vartheta(\widehat{x}, \xi, \lambda)=0$, while $\widehat{x}$ is not an efficient solution for $(P)$. Then, there exist a $x^{*} \in S$ and an index $k \in\{1,,,,, p\}$ such that:

$$
\left\{\begin{array}{l}
f_{i}\left(x^{*}\right) \leq f_{i}(\widehat{x}), \quad \forall i \in I \\
f_{k}\left(x^{*}\right)<f_{k}(\widehat{x})
\end{array}\right.
$$

Thus,

$$
\left\{\begin{array}{l}
\xi_{i}^{\prime}\left(x^{*}-\widehat{x}\right) \leq 0, \quad \forall i \in I \\
\xi_{k}^{\prime}\left(x^{*}-\widehat{x}\right)<0
\end{array}\right.
$$

From the latter inequalities and the assumption that $\lambda>0_{p}$, we get $\sum_{i=1}^{p} \lambda_{i} \xi_{i}^{\prime}(\widehat{x}-$ $\left.x^{*}\right)>0$. Hence, $\vartheta(\widehat{x}, \xi, \lambda)>0$, in contradiction with the assumption.

(ii) The proof is exactly the same as the one of Theorem 4 (ii). 
In Example 1, the strong KKT condition holds at $\widehat{x}=0$ as

$$
\text { ri } F_{*}(\widehat{x})+G_{*}(\widehat{x})=(-2,-1)+\mathbb{R}_{+}=(-2,+\infty) \ni 0 .
$$

Moreover, $\vartheta(\widehat{x}, \lambda)=0$ for all $\lambda>0_{2}$ by (18). Since the EADQ and the MOQ hold at $\widehat{x}$, we can assert that $\widehat{x}$ is an efficient solution for $(P)$ for by Theorems 7 and 8

In Example 3 we have seen that even the weak KKT condition fails at $\widehat{x}=0_{2}$ while $\vartheta(\widehat{x}, \lambda)=0$ for all $\lambda>0_{2}$ by (19). Concerning the data qualifications, EADQ holds but MOQ fails, Thus, no conclusion on the efficiency of $\widehat{x}$ can be obtained from the results in this section.

\section{Optimality conditions for isolated efficiency}

We say that the perturbed $K K T$ condition holds at $\widehat{x} \in S$ when there exists a positive scalar $\nu$ such that, for any vector $w \in \nu \mathbb{B}_{n}$, there exist scalars $\alpha_{i} \geq 0$ for $i \in I$ with $\sum_{i=1}^{p} \alpha_{i}=1$, a finite set $T^{*} \subseteq T(\widehat{x})$, and corresponding scalars $\beta_{t} \geq 0$ for $t \in T^{*}$, such that

$$
w \in \sum_{i=1}^{p} \alpha_{i} \partial f_{i}(\widehat{x})+\sum_{t \in T^{*}} \beta_{t} \partial g_{t}(\widehat{x}) .
$$

In geometric terms, the perturbed KKT condition holds at $\widehat{x} \in S$ if and only if

$$
0_{n} \in \operatorname{int}\left(F_{*}(\widehat{x})+G_{*}(\widehat{x})\right) .
$$

Theorem 9 (Perturbed KKT necessary condition under PMFCQ) Suppose that $\widehat{x}$ is an isolated efficient solution of $(P)$ with constant $\nu>0$ such that all constraint functions are continuously differentiable around $\widehat{x}$. Then, the following statements hold true:

(i) If the PMFCQ holds at $\widehat{x}$, then

$$
\nu \mathbb{B}_{n} \subseteq F_{*}(\widehat{x})+\bigcap_{\varepsilon>0} \overline{\operatorname{cone}}\left(\left\{\nabla g_{t}(\widehat{x}) \mid t \in T_{\varepsilon}(\widehat{x})\right\}\right) .
$$

(ii) If $(P)$ is continuous, and the MFCQ holds at $\widehat{x}$, then the perturbed KKT condition holds at $\widehat{x}$.

Proof (i) For each $x \in S$ we consider the DC (difference of convex) function $\varphi(x):=\max _{i \in I}\left\{f_{i}(x)-f_{i}(\widehat{x})\right\}-\nu\|x-\widehat{x}\|$. The definition of isolated efficiency means that $\varphi(x) \geq 0$ for all $x \in S$. Since $\varphi(\widehat{x})=0$, then $\widehat{x}$ is a minimizer of scalar optimization problem $\min _{x \in S} \varphi(x)$. Thus, using [1, Corollary 1], we obtain

$$
\partial(\nu\|\cdot-\widehat{x}\|)(\widehat{x}) \subseteq \partial\left(\max _{i \in I}\left\{f_{i}(\cdot)-f_{i}(\widehat{x})\right\}\right)(\widehat{x})+N(S, \widehat{x}) .
$$


On the other hand, from the well known rules of subdifferential calculus (see, e.g., [18, Section VI.4]) one has

$$
\partial(\nu\|\cdot-\widehat{x}\|)(\widehat{x})=\nu \mathbb{B}_{n} \quad \text { and } \quad \partial\left(\max _{i \in I}\left\{f_{i}(.)-f_{i}(\widehat{x})\right\}\right)(\widehat{x}) \subseteq F_{*}(\widehat{x}) .
$$

Combining (28), (29), the PMFCQ, and [27, Proposition 1 and Theorem 1], we get (27).

(ii) Observe that the continuity of $(P)$, together with 27, Proposition 2], implies the equivalence between the PMFCQ and the MFCQ. Now, invoking [27, Corollary 2] and taking (27) into account, we get $\nu \mathbb{B}_{n} \subseteq$ $\left(F_{*}(\widehat{x})+G_{*}(\widehat{x})\right)$, so that (26) holds. Hence, the perturbed KKT condition holds at $\widehat{x}$.

Theorem 10 (Perturbed KKT sufficient condition) If the perturbed $K K T$ condition holds at $\widehat{x} \in S$, then, $\widehat{x}$ is an isolated efficient solution for $(P)$.

Proof Let $x \in S \backslash\{\widehat{x}\}$. Since the perturbed KKT condition holds at $\widehat{x}$, there exists $\nu>0$ such that $\nu \mathbb{B}_{n} \subseteq \operatorname{int}\left(F_{*}(\widehat{x})+G_{*}(\widehat{x})\right)$. Recalling (26), and since $\frac{x-\widehat{x}}{\|x-\widehat{x}\|} \in \mathbb{B}_{n}$, there exist $\alpha_{i} \geq 0$ and $\xi_{i} \in \partial f_{i}(\widehat{x})$, for $i \in I$ with $\sum_{i=1}^{p} \alpha_{i}=1$, and $T^{*} \subseteq T(\widehat{x})$ with $\left|T^{*}\right|<\infty, \zeta_{t} \in \partial g_{t}(\widehat{x})$ and $\beta_{t} \geq 0$, for $t \in T^{*}$, such that

$$
\nu \frac{x-\widehat{x}}{\|x-\widehat{x}\|}=\sum_{i=1}^{p} \alpha_{i} \xi_{i}+\sum_{t \in T^{*}} \beta_{t} \zeta_{t}(\widehat{x})
$$

Multiplying both members of (30) by $x-\widehat{x}$, one gets

$$
\begin{aligned}
\nu\|x-\widehat{x}\| & =\sum_{i=1}^{p} \alpha_{i} \xi_{i}^{\prime}(x-\widehat{x})+\sum_{t \in T^{*}} \beta_{t} \zeta_{t}^{\prime}(x-\widehat{x}) \\
& \leq \sum_{i=1}^{p} \alpha_{i}\left(f_{i}(x)-f_{i}(\widehat{x})\right)+\sum_{t \in T^{*}} \beta_{t}\left(g_{t}(x)-g_{t}(\widehat{x})\right) \\
& \leq \sum_{i=1}^{p} \alpha_{i}\left(f_{i}(x)-f_{i}(\widehat{x})\right) \\
& \leq \max _{i \in I}\left\{f_{i}(x)-f_{i}(\widehat{x})\right\},
\end{aligned}
$$

where the last inequality holds as $\sum_{i=1}^{p} \alpha_{i}=1$. This shows $\widehat{x}$ is an isolated efficient solution for $(P)$.

In Example 1, $F_{*}(\widehat{x})+G_{*}(\widehat{x})=[-2,-1]+\mathbb{R}_{+}=[-2,+\infty)$, which is certainly a neighborhood of 0 , so that the perturbed KKT condition holds at $\widehat{x}$ with constant $\nu=2$. Then, $\widehat{x}$ is an isolated efficient solution by Theorem 10. In fact, $\max _{i \in I}\left\{f_{i}(x)-f_{i}(\widehat{x})\right\}=-2 x \geq 2|x|$ for all $x \in S=-\mathbb{R}_{+}$.

In Example 3. $F_{*}(\widehat{x})+G_{*}(\widehat{x})=\left\{x \in \mathbb{R}^{2} \mid x_{1} \geq-1, x_{2}>0\right\} \cup\{(-1,0)\}$, which is not a neighborhood of $\widehat{x}=0_{2}$. Thus, the perturbed KKT condition fails at $\widehat{x}$, but we cannot conclude from Theorem 9 that $\widehat{x}$ is not an isolated 
efficient solution as $(P)$ is not continuous and MFCQ fails, i.e., we are in a dubious case. Actually, $\max _{i \in I}\left\{f_{i}(x)-f_{i}(\widehat{x})\right\}=-x_{1} \geq\|x\|$ for all $x \in S=-\mathbb{R}_{+}^{2}$, i.e., $\widehat{x}$ is an isolated efficient solution too.

The final result in this paper requires $(P)$ be continuous, so that it cannot be applied to the above examples.

Theorem 11 (Characterization under MFCQ via gap function) Let $(P)$ be a continuous problem and $\widehat{x} \in S$ be such that all constraint functions are continuously differentiable around $\widehat{x}$ and $M F C Q$ holds. Then, $\widehat{x}$ is an isolated efficient solution if and only if there exists $\nu>0$ such that for all $w \in \nu \mathbb{B}_{n}$ there exist $\xi \in \prod_{i=1}^{p} \partial f_{i}(\widehat{x})$ and $\lambda \geq 0_{p}$ such that $\vartheta(\widehat{x}, \xi-w, \lambda)=0$.

Proof Consider the parametric convex MOSIO problem, with parameter $w \in \mathbb{R}^{n}$,

$\left(P_{w}\right) \quad$ minimize $\left(f_{1}(x)-w, \ldots, f_{p}(x)-w\right)$ subject to $g_{t}(x) \leq 0, t \in T$,

whose gap function is $\vartheta_{w}(x, \eta, \lambda)=\sup _{y \in S}\left\{\sum_{i=1}^{p} \lambda_{i} \eta_{i}^{\prime}(x-y)\right\}$, with $\eta_{i} \in$ $\partial\left(f_{i}-w\right)(\widehat{x})=\partial f_{i}(\widehat{x})-w$. The change of variables $\xi_{i}=\eta_{i}+w$, with $\xi_{i} \in \partial f_{i}(\widehat{x}), i \in I$, yields the identity $\vartheta_{w}(x, \eta, \lambda)=\vartheta(\widehat{x}, \xi-w, \lambda)$. We claim the equivalence between the following statements:

(i) $\widehat{x}$ is an isolated efficient solution of $(P)$.

(ii) For any $w \in \nu \mathbb{B}_{n}$, there exist scalars $\alpha_{i} \geq 0$ for $i \in I$ with $\sum_{i=1}^{p} \alpha_{i}=1$, a finite set $T^{*} \subseteq T(\widehat{x})$, and corresponding scalars $\beta_{t} \geq 0$ for $t \in T^{*}$, such that

$$
0_{n} \in \sum_{i=1}^{p} \alpha_{i} \partial\left(f_{i}-w\right)(\widehat{x})+\sum_{t \in T^{*}} \beta_{t} \partial g_{t}(\widehat{x}) .
$$

(iii) $\widehat{x}$ is a weak efficient solution of $\left(P_{w}\right)$ for all $w \in \nu \mathbb{B}_{n}$.

(iv) For any $w \in \nu \mathbb{B}_{n}$, there exist $\xi \in \prod_{i=1}^{p} \partial f_{i}(x)$ and $\lambda \in \Delta_{+}^{p}$ such that $\vartheta(\widehat{x}, \xi-w, \lambda)=0$.

$[(i) \Leftrightarrow(i i)]$ It follows from Theorems 9 and 10 as $(i i)$ is a reformulation of the perturbed KKT condition for $(P) .[(i i) \Leftrightarrow(i i i)]$ It follows from Corollary 1, applied to $\left(P_{w}\right)$, taking into account that LFMCQ holds at $\widehat{x}$ by Theorem 1 (the constraints of $\left(P_{w}\right)$ and $(P)$ coincide).

$[(i i i) \Leftrightarrow(i v)]$ It follows from Theorem 4 applied to $\left(P_{w}\right)$.

\section{References}

1. Amahroq, T., Penot, J.-P., Say, A: On the subdifferentiability of the difference of two functions and local minimization. Set-Valued Anal. 16, 413-427 (2008)

2. Auslender, A.: Optimisation: Méthodes Numériques. Masson, Paris (1976)

3. Auslender, A.: Stability in mathematical programming with nondifferentiable. data. SIAM J. Control Optim. 22, 239-254 (1984)

4. Caristi, G., Ferrara, M., Stefanescu, A.: Semi-infinite multiobjective programming with generalized invexity. Math. Reports 12, 217-233 (2010) 
5. Chen, C.Y., Goh, C.J., Yang, X.Q.: The gap function of a convex multicriteria optimization problem. European J. Oper. Res. 111, 142-151 (1998)

6. Chuong, T.D., Kim, D.S.: Nonsmooth semi-infinite multiobjective optimization problems. J. Optim. Theory Appl. 160, 748-762 (2014)

7. Chuong, T.D., Yao, J.-Ch.: Isolated and proper efficiencies in semi-infinite vector optimization problems. J. Optim. Theory Appl. 162, 447-462 (2014)

8. Dinh, N., Goberna, M.A., Long, D.H., López, M.A.: Characterizing weak solutions for vector optimization problems. arXiv:1602.03367

9. Dumford, N., Schwartz, J.T.: Linear Operators: General Theory. Wiley, Chichester (1988)

10. Ginchev, I., Guerraggio, A., Rocca, M.: From scalar to vector optimization. Appl. Math. 51, 618-630 (2006)

11. Goberna, M.A., Gómez, S., Guerra-Vazquez, Todorov, M.I.: Sensitivity analysis in linear semi-infinite programming: perturbing cost and right-hand-side coefficients. European J. Oper. Res. 181, 1069-1085 (2007)

12. Goberna, M.A., Guerra-Vazquez, F., Todorov, M.I.: Constraint qualifications in linear vector semi-infinite optimization. European J. Oper. Res. 227, 12-21 (2013)

13. Goberna, M.A., Guerra-Vazquez, Todorov, M. I.: Constraint qualifications in convex vector semi-infinite optimization. European J. Oper. Res. 249, 32-40 (2016)

14. Goberna, M.A., López, M.A.: Linear Semi-Infinite Optimization. Wiley, Chichester (1998)

15. Goberna, M.A., Terlaky, T., Todorov, M.I.: Sensitivity analysis in linear semiinfinite programming via partitions. Math. Oper. Res. 35, 14-25 (2010).

16. Guerra-Vazquez, F., Rückmann, J.-J.: On proper efficiency in multiobjective semi-infinite optimization. In Optimization and Control Techniques and Applications (H. Xu, K.L. Teo, Y. Zhang, eds.), Springer, Berlin, pp. 115-135 (2014).

17. Hearn, D.W.: The gap function of a convex program. O. R. Letters 1, 67-71 (1992)

18. Hiriart-Urruty, J.-B., Lemaréchal, C.: Convex Analysis and Minimization Algorithms, I. Springer, Berlin-Heidelberg (1991)

19. Jongen, H.T., Twilt, F., Weber, G.W.: Semi-infinite optimization: structure and stability of feasible set. J. Optim. Theory Appl. 72, 529-552 (1992)

20. Kanzi, N.: Constraint qualifications in semi-infinite systems and their applications in nonsmooth semi-infinite problems with mixed constraints. SIAM J. Optim. 24, 559-572 (2014)

21. Kanzi, N.: On Strong KKT Optimality conditions for multiobjective semiinfinite programming problems with Lipschitzian data. Optim. Letters 9, 1121-1129 (2015)

22. Kanzi, N., Soleymani-damaneh, M.: Slater CQ, optimality and duality for quasiconvex semi-infinite optimization problems. J. Math. Anal. Appl. 434, 638-351 (2016)

23. Kanzi, N., Nobakhtian, S.: Optimality conditions for nonsmooth semi-infinite multiobjective programming. Optim. Letters 8, 1517-1528 (2014)

24. Kuroiwa, D, Lee, G. M.: On robust convex multiobjective optimization. J. Nonlinear Convex Anal. 15, 1125-1136 (2014)

25. Li, W., Nahak, C., Singer, I.: Constraint qualifications in semi-infinite systems of convex inequalities. SIAM J. Optim. 11, 31-52 (2000) 
26. Maeda, T.: Constraint qualifications in multiobjective optimization problems: differentiable case. J. Optim. Theory Appl. 80, 483-500 (1994)

27. Mordukhovich, B.S., Nghia, T.T.A.: Constraint qualifications and optimality conditions for nonconvex semi-infinite and infinite programs. Math. Programming 139B, 271-300 (2013)

28. Rockafellar, R.T.: Convex Analysis. Princeton U. P., Princeton (1970)

29. Sarabi, M.E., Soleimani-damaneh, M.: Revisiting the function of a multicriteria optimization problem. Int. J. Comput Math. 86, 860-863 (2009)

30. Soleimani-damaneh, M.: The gap function for optimization problems in Banach spaces. Nonlinear Anal. 69, 716-723 (2008)

31. Soleimani-damaneh, M.: Nonsmooth optimization using Mordukhovich subdifferential. SIAM J. Control Optim. 48, 3403-3432 (2010)

32. Tanino, T.: Conjugate duality in vector optimization. J. Math. Anal. Appl. 167, 84-97 (1992)

33. Zalinescu, C.: Convex Analysis in General Vector Spaces. World Scientific, Singapore (2002) 